\title{
Minimal Critical Sets of Refined Inertias for Irreducible Sign Patterns of Order 2
}

\author{
Ber-Lin Yu \\ Faculty of Mathematics and Physics, Huaiyin Institute of Technology, Huai'an, China \\ Email: berlinyu@163.com
}

Received May 1, 2013; revised June 1, 2013; accepted June 8, 2013

Copyright (C) 2013 Ber-Lin Yu. This is an open access article distributed under the Creative Commons Attribution License, which permits unrestricted use, distribution, and reproduction in any medium, provided the original work is properly cited.

\begin{abstract}
Let $S$ be a nonempty, proper subset of all refined inertias. Then, $S$ is called a critical set of refined inertias for ireducible sign patterns of order $n$ if $S \subseteq r i(A)$ is sufficient for any sign pattern $A$ to be refined inertially arbitrary. If no proper subset of $S$ is a critical set of refined inertias, then $S$ is a minimal critical set of refined inertias for sign patterns of order $n$. In this paper, all minimal critical sets of refined inertias for irreducible sign patterns of order 2 are identified. As a by-product, a new approach is presented to identify all minimal critical sets of inertias for irreducible sign patterns of order 2.
\end{abstract}

Keywords: Associated Digraph; Inertially Arbitrary Sign Pattern; Refined Inertia; Critical Set of Refined Inertias

\section{Introduction}

A $n \times n$ sign pattern is a matrix $\hat{A}=\left[\alpha_{i j}\right]$ with entries from the set $\{+,-, 0\}$ where + (respectively, - ) denotes a positive (respectively, negative) real number; see, e.g., [1]. The set of all real matrices with the same sign pattern as the $n \times n$ sign pattern $\hat{A}$ is the qualitative class

$$
Q(\hat{A})=\left\{A=\left[a_{i j}\right] \in M_{n}(R) \mid \operatorname{sign}\left(a_{i j}\right)=\alpha_{i j}\right\} .
$$

A subpattern of an $n \times n$ sign pattern $\hat{A}$ is a sign pattern $\hat{B}$ obtained by replacing some (possibly empty) subset of the nonzero entries of $\hat{A}$ with zeros. If $\hat{B}$ is a subpattern of $\hat{A}$, then $\hat{A}$ is a superpattern of $\hat{B}$. Two square zero-nonzero patterns $\hat{A}$ and $\hat{B}$ are equivalent if one can be obtained from the other by any combination of transposition and permutation similarity. A sign pattern $\hat{A}$ is reducible if it is permutation similar to a pattern of the form

$$
\left(\begin{array}{cc}
\hat{A}_{11} & \hat{A}_{12} \\
0 & \hat{A}_{22}
\end{array}\right)
$$

where $\hat{A}_{11}, \hat{A}_{22}$ are square and non-vacuous. A pattern is irreducible if it is not reducible.

The inertia of a matrix $A$ is an ordered triple $\left(n_{+}, n_{-}, n_{0}\right)$ where $n_{+}, n_{-}$and $n_{0}$ are the number of eigenvalues of $A$ with positive, negative and zero real part, respectively; see, e.g., [2]. The refined inertia of $A$ is the ordered quadruple $\left(n_{+}, n_{-}, n_{z}, 2 n_{p}\right)$ of nonnegative integers that sum to $n$, where $\left(n_{+}, n_{-}, n_{z}+2 n_{p}\right)$ is the inertia of $A$ while $n_{z}$ is the number of 0 as an eigenvalue of $A$ and $2 n_{p}$ is the number of eigenvalues of $A$ with nonzero pure imaginary eigenvalues; see, e.g., [3]. The inertia (respectively, refined inertia) of a sign pattern $\hat{A}$ is $i(\hat{A})=\{i(A) \mid A \in Q(\hat{A})\}$ (respectively, $r i(\hat{A})=\{r i(A) \mid A \in Q(\hat{A})\})$. A $n \times n$ sign pattern $\hat{A}$ is an inertially arbitrary pattern (IAP) if given any ordered triple $\left(n_{+}, n_{-}, n_{0}\right)$ of nonnegative integers with $n_{+}+n_{-}+n_{0}=n$, there exists a real matrix $A \in Q(\hat{A})$ such that $i(A)=\left(n_{+}, n_{-}, n_{0}\right)$; see, e.g., $[4,5]$ and the reference therein. Similarly, $\hat{A}$ is a refined inertially arbitrary pattern (rIAP) if given any ordered quadruple $\left(n_{+}, n_{-}, n_{z}, 2 n_{p}\right)$ of nonnegative integers that sum to $n$, there exists a real matrix $A \in Q(\hat{A})$ such that $\operatorname{ri}(A)=\left(n_{+}, n_{-}, n_{z}, 2 n_{p}\right)$; see, e.g., [3].

Let $S$ be a nonempty, proper subset of the set of all inertias for any $n \times n$ zero-nonzero (or sign) pattern $\hat{A}$. If $S \subseteq i(\hat{A})$ is sufficient for $\hat{A}$ to be inertially arbitrary, then $S$ is said to be a critical set of inertias for zero-nonzero (or sign) patterns of order $n$ and if no proper subset of $S$ is a critical set of inertias, then $S$ is said to be a minimal critical set of inertias for zero- 
nonzero (or sign) patterns of order $n$; see, e.g., [6]. Critical sets of refined inertias for irreducible zero-nonzero patterns are defined in [7]. Similarly, we introduce the concept of a critical set of refined inertias for sign patterns. Let $S$ be a nonempty, proper subset of all refined inertias. Then, $S$ is called a critical set of refined inertias for sign patterns of order $n$, if the set $S \subseteq \operatorname{ri}(\hat{A})$ is sufficient for any sign pattern $\hat{A}$ to be refined inertially arbitrary. If no proper subset of $S$ is a critical set of refined inertias, then $S$ is a minimal critical set of refined inertias for irreducible sign patterns of order $n$. We note that all minimal critical sets of inertias for irreducible sign patterns of order 2 have been identified in [6]. But, identifying all minimal critical sets of inertias for irreducible zero-nonzero (or sign) patterns of order $\geq 3$ has been posed as an open question in [6]. Also open is the minimum cardinality of such a set. The concept of critical sets of refined inertias for sign pattern is introduced for the first time. In this work, we concentrate on the minimal critical sets of refined inertias for irreducible sign patterns of order 2 .

Our work is organized as follows. Section 2 describes some preliminary results on the refined inertias of sign patterns. The minimal critical sets of refined inertias for irreducible sign patterns of order 2 are identified in Section 3. In Section 4, as a by-product, an alternative proof is given to identify all minimal critical sets of inertias for $2 \times 2$ irreducible sign patterns. Some concluding remarks are given in Section 5.

\section{Preliminaries}

Recall that a sign pattern $\hat{A}=\left[\alpha_{i j}\right]$ has its associated digraph $D(\hat{A})$ with vertex set $\{1,2, \cdots, n\}$ and for all $i$ and $j$, a positive (resp., negative ) arc from $i$ to $j$ if and only if $\alpha_{i j}=+$ (resp., $\left.\alpha_{i j}=-\right)$. A (directed) simple cycle (or a $k$-cycle) $\gamma$ of length $k$ is a sequence of $k$ arcs $\left(i_{1}, i_{2}\right),\left(i_{2}, i_{3}\right), \cdots,\left(i_{k}, i_{1}\right)$ such that these vertices are distinct; see, e.g., [1]. The sign positive (or negative) of a simple cycle in a sign pattern $\hat{A}$ is the actual product of the entries in the cycle, following the obvious rules that multiplication is commutative and associative, and $(+)(+)=+,(+)(-)=-$.

Lemma 2.1. let $\hat{A}$ be an irreducible sign pattern of order 2. Then the following are equivalent:

(1) $\hat{A}$ is spectrally arbitrary;

(2) $\hat{A}$ is inertially arbitrary;

(3) Up to equivalence, $\hat{A}=\left(\begin{array}{ll}+ & + \\ - & -\end{array}\right)$;

(4) Associated digraph of $\hat{A}, D(\hat{A})$, has two loops of opposite sign and a negative 2-cycle.

Proof. The equivalences of $(1) \Leftrightarrow(2) \Leftrightarrow(3)$ follow from Proposition 3 in [6]. The equivalence of $(3) \Leftrightarrow(4)$ can be verified directly.

It is known that there are seven refined inertias for $2 \times 2$ sign patterns. To identify all minimal critical sets of refined inertias for irreducible sign patterns of order 2 , the following three sign patterns are necessary to be investigated.

Lemma 2.2. Let $M=\left(\begin{array}{ll}- & + \\ + & -\end{array}\right)$. Then, $M$ allows the only refined inertias $(0,1,1,0),(0,2,0,0)$ and $(1,1,0$, $0)$.

Proof. Since $M$ requires every realization with a negative trace, the refined inertias $(2,0,0,0),(0,0,2,0)$, $(0,0,0,2)$ and $(1,0,1,0)$ cannot be allowed by $M$. For the remaining refined inertias, consider realizations of $M,\left(\begin{array}{cc}-1 & 1 \\ 1 & -1\end{array}\right),\left(\begin{array}{cc}-1 & 1 / 2 \\ 1 / 2 & -1\end{array}\right),\left(\begin{array}{cc}-1 & 2 \\ 2 & -1\end{array}\right)$ with refined inertias $(0,1,1,0),(0,2,0,0)$ and $(1,1,0,0)$, respectively. It follows that $M$ allows the only refined inertias $(0,1,1$, $0),(0,2,0,0),(1,1,0,0)$.

Lemma 2.3. Let $N=\left(\begin{array}{l}+ \\ + \\ +\end{array}\right)$. Then, $N$ allows all refined inertias except $(0,1,1,0),(0,2,0,0),(0,0,2,0)$ and $(0,0,0,2)$.

Proof. Since $N$ requires every realization with $a$ positive trace, the refined inertias $(0,1,1,0),(0,2,0,0)$, $(0,0,2,0)$ and $(0,0,0,2)$ cannot be allowed by $N$. Consider the following realizations of $N$,

$\left(\begin{array}{cc}1 & 1 / 2 \\ 1 / 2 & 1\end{array}\right),\left(\begin{array}{ll}1 & 1 \\ 1 & 1\end{array}\right)$ and $\left(\begin{array}{ll}1 & 1 \\ 4 & 1\end{array}\right)$ with refined inertias $(2$, $0,0,0),(1,0,1,0)$ and $(1,1,0,0)$, respectively. It follows that $N$ allows all refined inertias except $(0,1,1$, $0),(0,2,0,0),(0,0,2,0)$ and $(0,0,0,2)$.

Lemma 2.4. Let $P=\left(\begin{array}{cc}0 & + \\ - & 0\end{array}\right)$. Then, $P$ allows the refined inertia $(0,0,0,2)$.

Proof. Lemma 2.4 follows from the fact that a realization of $P,\left(\begin{array}{cc}0 & 1 \\ -1 & 0\end{array}\right)$, has $(0,0,0,2)$ as its refined inertia.

\section{Minimal Critical Sets of Refined Inertias for Irreducible Sign Patterns of Order 2}

We are now to identify all minimal critical sets of refined inertias for irreducible sign patterns of order 2 .

Theorem 3.1. The set $\{(0,0,2,0)\}$ is the only minimal critical set with a single refined inertia for $2 \times 2$ ireducible sign patterns.

Proof. Lemma 2.2 indicates that $\{(0,2,0,0),\{(1,1,0$, $0)\}$ and $\{(0,1,1,0)\}$ cannot be a minimal critical set of refined inertias. Lemma 2.3 indicates that $\{(2,0,0,0)\}$ and $\{(1,0,1,0)\}$ cannot be a minimal critical set of re- 
fined inertias. Lemma 2.4 indicates that $\{(0,0,0,2)\}$ cannot be a minimal critical set of refined inertias. So, it suffices to show that the set $\{(0,0,2,0)\}$ is a minimal critical sets of refined inertias.

If $\{(0,0,2,0)\}$ is allowed by an arbitrary irreducible sign pattern $\hat{A}$ of order 2 , then all the main diagonal entries of $\hat{A}$ must be nonzero. Since $\hat{A}$ allows a realization with a zero trace, the two diagonal entries of $\hat{A}$ are of opposite sign. That is to say, the associated digraph $D(\hat{A})$ of $\hat{A}$, has a positive loop and a negative loop. For $\hat{A}$ allows a realization with zero determinant, $D(\hat{A})$ has a negative 2-cycle. It follows from Lemma 2.1 that $\hat{A}$ is refined inertially arbitrary.

Theorem 3.2. The refined inertia sets $\{(0,0,0,2),(1$, $0,1,0)\},\{(0,0,0,2),(0,1,1,0)\},\{(0,0,0,2),(2,0,0$, $0)\},\{(0,0,0,2),(0,2,0,0)\},\{(0,0,0,2),(1,1,0,0)\}$, $\{(1,0,1,0),(0,1,1,0)\},\{(1,0,1,0),(0,2,0,0)\},\{(1,0$, $1,0),(2,0,0,0)\}$ and $\{(2,0,0,0),(0,2,0,0)\}$ are minimal critical sets of refined inertias for irreducible sign patterns of order 2 .

Proof. Let $\hat{A}$ be an arbitrary irreducible sign pattern of order 2 . If $\{(0,0,0,2),(1,0,1,0)\} \subseteq r i(\hat{A})$, then $\hat{A}$ allows a realization with a positive trace and a realization with zero trace. It follows that $D(\hat{A})$ has a positive loop and a negative loop. Since $\hat{A}$ allows a realization with zero determinant, $D(\hat{A})$ has a negative 2-cycle. By Lemma $2.1, \hat{A}$ is refined inertially arbitrary and $\{(0$, $0,0,2),(1,0,1,0)\}$ is a minimal critical set of refined inertias for $2 \times 2$ irreducible sign patterns. Similarly, we can show the refined inertias $\{(0,0,0,2),(0,1,0)\}$, $\{(0,0,0,2),(2,0,0,0)\},\{(0,0,0,2),(0,2,0,0)\},\{(1,0$, $1,0),(0,1,1,0)\},\{(1,0,1,0),(0,2,0,0)\},\{(1,0,1,0)$, $(2,0,0,0)\}$ and $\{(2,0,0,0),(0,2,0,0)\}$ are minimal critical sets of refined inertias for $2 \times 2$ irreducible sign patterns.

For the refined inertia set $\{(0,0,0,2),(1,1,0,0)\}$ $\subseteq \operatorname{ri}(\hat{A})$, we claim that the diagonal entries of $\hat{A}$ must be nonzero. In fact, assume that there exists at least a zero diagonal entry of $\hat{A}$. Then sign pattern $\hat{A}$ requires nonsingularity. It is contradicted that $\hat{A}$ allows two realizations with a positive and negative determinant, respectively. So, the diagonal entries of $\hat{A}$ must be nonzero. And the fact that the diagonal entries of $\hat{A}$ are of opposite sign follows from that $\hat{A}$ allows a realization with a zero trace. $D(\hat{A})$ has a negative simple cycle of length 2 follows from that the inertia $(0,0,0,2)$ $\in \operatorname{ri}(\hat{A})$.

Next we identify all minimal critical sets of refined inertias for $2 \times 2$ irreducible sign patterns.

Theorem 3.3. The sets $\{(0,0,2,0)\},\{(0,0,0,2),(1,0$, $1,0)\},\{(0,0,0,2),(0,1,1,0)\},\{(0,0,0,2),(2,0,0,0)\}$, $\{(0,0,0,2),(0,2,0,0)\},\{(0,0,0,2),(1,1,0,0)\},\{(1,0$, $1,0),(0,1,1,0)\},\{(1,0,1,0),(0,2,0,0)\},\{(1,0,1,0)$, $(2,0,0,0)\}$ and $\{(2,0,0,0),(0,2,0,0)\}$ are the only minimal critical sets of refined inertias for $2 \times 2$ irreducible sign patterns.

Proof. By Theorems 3.1 and 3.2, the refined inertia sets stated in Theorem 3.3 are minimal critical sets of refined inertias for $2 \times 2$ irreducible sign patterns. To show there exists no other minimal critical sets of refined inertias, it suffices to show that the remaining nine refined inertia sets with cardinality $2,\{(1,0,1,0),(2,0,0$, $0)\},\{(1,0,1,0),(1,1,0,0)\},\{(2,0,0,0),(1,1,0,0)\}$, $\{(0,1,1,0),(0,2,0,0)\},\{(0,1,1,0),(1,1,0,0)\},\{(1,1$, $0,0),(0,2,0,0)\}$ and the two refined inertia sets with cardinality $3,\{(1,0,1,0),(2,0,0,0),(1,1,0,0)\}$ and $\{(0,1,1,0),(0,2,0,0),(1,1,0,0)\}$ are not critical sets of refined inertias. By Lemma 2.3, $\{(1,0,1,0),(2,0,0$, $0)\},\{(1,0,1,0),(1,1,0,0)\},\{(2,0,0,0),(1,1,0,0)\}$ and $\{(1,0,1,0),(2,0,0,0)\},(1,1,0,0)\}$ are not critical sets of refined inertias. By Lemma 2.2, $\{(0,1,1,0),(0,2$, $0,0)\},\{(0,1,1,0),(1,1,0,0)\},\{(1,1,0,0),(0,2,0,0)\}$ and $\{(0,1,1,0),(0,2,0,0)\},(1,1,0,0)\}$ are not critical sets of refined inertias.

The following theorem follows directly for Theorem 3.3.

Theorem 3.4. Let $\hat{A}$ be a irreducible sign pattern of order 2 . Then the following are equivalent:

1) $\hat{A}$ is refined inertially arbitrary;

2) $\hat{A}$ allows $(0,0,2,0)$;

3) $\hat{A}$ allows $(0,0,0,2)$ and $(1,0,1,0)$;

4) $\hat{A}$ allows $(0,0,0,2)$ and $(0,1,1,0)$;

5) $\hat{A}$ allows $(0,0,0,2)$ and $(2,0,0,0)$;

6) A allows $(0,0,0,2)$ and $(0,2,0,0)$;

7) $\hat{A}$ allows $(0,0,0,2)$ and $(1,1,0,0)$;

8) $\hat{A}$ allows $(1,0,1,0)$ and $(0,1,1,0)$;

9) $\hat{A}$ allows $(1,0,1,0)$ and $(0,2,0,0)$;

10) $\hat{A}$ allows $(1,0,1,0)$ and $(2,0,0,0)$;

11) $\hat{A}$ allows $(2,0,0,0)$ and $(0,2,0,0)$.

\section{Minimal Critical Sets of Inertias for Irreducible Sign Patterns of Order 2}

In [6], all minimal critical sets of inertias for $2 \times 2$ irreducible sign patterns, which are restated here as Theorem 4.1, have been identified. In this section, we present an alternative proof in terms of critial sets of refined inertias, as a by-product.

Theorem 4.1. The sets $\{(2,0,0),(0,0,2)\},\{(0,2,0)$, $(0,0,2)\},\{(2,0,0),(0,1,1)\},\{(0,2,0),(1,0,1)\},\{(1,1$, $0),(0,0,2)\},\{(1,0,1),(0,0,2)\},\{(0,1,1),(0,0,2)\}$, $\{(2,0,0),(0,2,0)\}$ and $\{(1,0,1),(0,1,1)\}$ are the only minimal critical sets of inertias for $2 \times 2$ irreducible sign patterns.

Proof. Note that an irreducible $2 \times 2$ sign pattern: allows $\{(0,0,2),(2,0,0)\}$ if and only if it allows $\{(2,0,0,0),(0,0,2,0)\}$ or $\{(2,0,0,0),(0,0,0,2)\}$, allows $\{(0,0,2),(0,2,0)\}$ if and only if it allows 
$\{(0,2,0,0),(0,0,2,0)\}$ or $\{(0,2,0,0),(0,0,0,2)\}$, allows $\{(2,0,0),(0,1,1)\}$ if and only if it allows $\{(2,0,0,0),(0,1,1,0)\}$, allows $\{(0,2,0),(1,0,1)\}$ if and only if it allows $\{(0,2,0,0),(1,0,1,0)\}$, allows $\{(1,1,0),(0,0,2)\}$ if and only if it allows $\{(0,0,0,2),(1,1,0,0)\}$ or $\{(0,0,2,0),(1,1,0,0)\}$, allows $\{(1,0,1),(0,0,2)\}$ if and only if it allows $\{(0,0,0,2),(1,0,1,0)\}$ or $\{(0,0,2,0),(1,0,1,0)\}$, allows $\{(0,1,1),(0,0,2)\}$ if and only if it allows $\{(0,0,0,2),(0,1,1,0)\}$ or $\{(0,0,2,0),(0,1,1,0)\}$, allows $\{(2,0,0),(0,2,0)\}$ if and only if it allows $\{(2,0,0,0),(0,2,0,0)\}$, allows $\{(1,0,1),(0,1,1)\}$ if and only if it allows $\{(0,1,1,0),(1,0,1,0)\}$.

Since all of the refined inertia sets above are critical sets of refined inertias (not necessarily minimal), the above sets of inertias are critical sets, and indeed are also easily seen to be minimal (since at least one of the corresponding sets of refined inertias is minimal in each case). It follows that the above sets of inertias are the only minimal critical sets of inertias because the corresponding sets of refined inertias are the only minimal critical sets of refined inertias.

\section{Concluding Remarks}

We have identified all minimal critical sets of refined inertias for irreducible sign patterns of order 2. As a by-product, all minimal critical sets of inertias for irreducible sign patterns of order 2 have also been identified in a new proof. In a follow-up paper, we will consider other cases, e.g., $n=3$, though identification of all critical sets of inertias for irreducible sign patterns of order $n \geq 3$ has been posed as an open question in [6].

\section{Acknowledgements}

The authors would like to express their great gratitude to the referees and editor for their constructive comments and suggestions that led to the enhancement of this paper. This research was supported in part by the Sci. \& Tech. Research Fund of Huaiyin Institute of Technology (HGB1111), National Natural Science Foundation of China (11201168) and Natural Science Foundation of the Higher Education Institutions of Jiangsu Province (Grant No. 12KJB110001).

\section{REFERENCES}

[1] F. Hall and Z. Li, "Sign Pattern Matrices," In: L. Hogben, Ed., Handbook of Linear Algebra, Chapman \& Hall/CRC Press, Boca Ration, 2007.

[2] R. A. Horn and C. R. Johnson, "Matrix Analysis," Cambridge University Press, New York, 1985. doi:10.1017/CBO9780511810817

[3] L. Deaett, D. D. Olesky and P. van den Driessche, "Refined Inertially and Spectrally Arbitrary Zero-Nonzero Patterns," The Electronic Journal of Linear Algebra, Vol. 20, 2010, pp. 449-467.

[4] M. S. Cavers and K. N. Vander Meulen. "Spectrally and Inertially Arbitrary Sign Patterns," Linear Algebra and Its Applications, Vol. 394, No. 1, 2005, pp. 53-72. doi:10.1016/j.laa.2004.06.003

[5] M. S. Cavers, K. N. Meulen and L. Vanderspek. "Sparse Inertially Arbitrary Patterns," Linear Algebra and Its Applications, Vol. 431, No. 11, 2009, pp. 2024-2034. doi:10.1016/j.laa.2009.06.040

[6] I. J. Kim, D. D. Olesky and P. van den Driessche, "Critical Sets of Inertias for Matrix Patterns," Linear and Multilinear Algebra, Vol. 57, No. 3, 2009, pp. 293-306. doi:10.1080/03081080701616672

[7] B. L. Yu, T. Z. Huang, J. Luo and H. B. Hua, "Critical Sets of Refined Inertias for Irreducible Zero-Nonzero Patterns of Orders 2 and 3," Linear Algebra and Its Applications, Vol. 437, No. 2, 2012, pp. 490-498. doi:10.1016/j.laa.2012.03.007 